\title{
The Effect of Decision Satisfaction Prediction in Argumentation-Based Negotiation
}

\author{
João Carneiro ${ }^{1,2}$, Diogo Martinho ${ }^{1}$, Goreti Marreiros ${ }^{1}$, and Paulo Novais ${ }^{2}$ \\ ${ }^{1}$ GECAD - Knowledge Engineering and Decision Support Group, Institute of Engineering - \\ Polytechnic of Porto, Porto, Portugal \\ \{jomrc, 1090557, mgt\} @isep.ipp.pt \\ ${ }^{2}$ ALGORITMI Centre, at University of Minho, Braga, Portugal \\ pjon@di.uminho.pt
}

\begin{abstract}
Supporting group decision-making is a complex process, especially when decision-makers have no opportunity to gather at the same place and at the same time. Besides that, finding solutions may be difficult in case representing agents are not able to understand the process and support the decision-maker accordingly. Here we propose a model and an algorithm that will allow the agent to analyse tendencies. This way we intend that agents can achieve decisions with more quality and with higher levels of consensus. Our model allows the agent to redefine his objectives to maximize both his and group satisfaction. Our model proved that agents that use it will obtain higher average levels of consensus and satisfaction. Besides that, agents using this model will obtain those higher levels of consensus and satisfaction in most of the times compared to agents that do not use it.
\end{abstract}

Keywords: Group Decision Support Systems, Argumentation, Decision Satisfaction, Automatic Negotiation, Multi-Agent Systems

\section{$1 \quad$ Introduction}

The future and success of organizations depend greatly on the quality of every decision made. It is known that most of the decisions in organizations are made in-group [1]. To support this type of decision, the Group Decision Support Systems (GDSS) have been widely studied throughout the last decades [2, 3]. However, in the last ten/twenty years, we have seen a remarkable change in the context where the decision-making process happens, especially in large organizations $[4,5]$. With the appearance of global markets, the growth of multinational enterprises and a global vision of the planet, we easily find chief executive officers and top managers (decision-makers) spread around the world, in countries with different time zones. In order to provide an answer and operate correctly in this type of scenarios the traditional GDSS have evolved to what we identify today as Ubiquitous Group Decision Support Systems (UbiGDSS). The UbiGDSS support the decision-making process by using the main characteristics of ubiquity ("anytime and "anywhere") [6, 7]. 
There are some works in the literature that address the term of UbiGDSS [7]. The UbiGDSS may present different levels of complexity. They can provide information about decision-maker preferences and other simple statistical information [8]. They can also follow the entire decision-making process using agents that represent decisionmakers. These agents can use automatic negotiation models to solve problems, finding consensual alternatives that provide a high level of satisfaction $[9,10]$. However, most of published works address the decision in a completely different perspective. In literature, we find many proposed works that deal with the topic of decision-making through the use of agents, argumentation models, heuristics, etc. [11, 12]. However, the type of the decision and how it is oriented in those works is completely different from the decision-making context where organizations make use of UbiGDSS. For instance, in most of works in the literature that use agents to perform automatic negotiation tasks, they will be either fully competitive or fully collaborative. In order to support decisionmaking groups that represent an entire organization while using automatic negotiation mechanisms it is necessary to pay close attention to some details. The system will be used by humans, it is necessary to involve the decision-maker in the decision-making process, it is very important that the decision-maker understands the logic behind such suggestions and it is essential to find solutions which result from the exchange of knowledge and the creation of intelligence $[13,14]$. In this type of context, it is not the right approach to take advantage of the agent's lack of knowledge just to place him in a better position to accept a certain request. We are dealing with a context where there will be a combination of both competition and collaboration.

In this work, we study how the decision-making group can obtain higher levels of consensus and satisfaction by giving agents the ability to predict the final level of satisfaction, which theoretically should lead to decisions with more quality. For that, we propose a model and an algorithm that will allow agents to identify an alternative tendency and that will result in the agent redefining objectives and obtaining a higher level of satisfaction compared to the case where he does not make that redefinition.

The model is structured in two parts. In the first part, whenever the agent identifies an alternative tendency he will verify if new alternatives should be added to his objectives. In the second part, the agent will analyse and select the best alternative from his objectives to make a request at a certain time.

The hypotheses which we intend to analyse in this work are: (h1) intelligent agents are capable to understand the context and show flexibility to make better decisions, (h2) agents able to predict the final level of satisfaction make decisions easier (achieve higher level of consensus) and (h3) agents able to predict the final level of satisfaction make more satisfactory decisions (the perception of quality level of the agent is higher). To test the proposed model and algorithm we have used an argumentation model adapted to the context of this work and that has been introduced before. Several experiments were performed in two simulation environments with different levels of complexity. The goal was to compare agents with the ability to analyse tendencies with agents without the same ability. We have anticipated that agents able to analyse tendencies and redefine objectives, and therefore being more flexible, will be able to achieve decisions with more quality and with higher levels of consensus. 
The rest of the paper is organized as follows: in the next section our approach is presented, where the model and the algorithm are described. In the section 3 we present the evaluation done to our work and report the obtained results. Finally, some conclusions are taken in section 4 , along with the work to be done hereafter.

\section{Methods}

Being able to predict the final level of satisfaction may have a great impact on the final level of satisfaction of the decision-maker, the decision group and in the decision quality. We can only make such statement due to the relation existing between the satisfaction and the perception of the decision quality [15]. It is considered that the final satisfaction of a decision-maker or a group of decision-makers reflects the perception of quality and other things $[6,10]$. In order to measure satisfaction some aspects can be considered such as: the results, the process, the defined behaviour or the strategy towards a certain problem, the interactions, etc [10]. In order to predict satisfaction (in a human way) it is first necessary to have the sensitivity to do so and secondly it is necessary to have the knowledge about the context (which sometimes may not be possible).

As introduced in literature the satisfaction can be used as a metric (effective and efficient) to validate the quality of negotiation models, group decision support systems, etc [10]. It is important to note that the satisfaction is widely used in literature as a metric for many other things, such as: life satisfaction [16], job satisfaction [17], etc.

In many existing negotiation models, agents will send requests hoping that other agents will accept them. They use arguments that can justify requests and also persuade other agents $[18,19]$. Besides that, it is very common to see agents that use algorithms to identify moments when they can accept a certain request [20]. In this work, we study a new branch, which is the agent's ability to analyse tendencies, and how that will affect the final satisfaction and the ability to reach consensus. We will consider that a decision-maker, within a scale [0...1] will do the alternatives' appreciation of $\{[$ Alt1, 0.89], [Alt2, 0.54], [Alt3, 0.34], [Alt4, 0.11]\}. To simplify our scenario we assume that the final satisfaction level is equivalent to the appreciation done to the chosen alternative, which we have a type of meeting that selects the winning alternative with the highest level of consensus after 10 rounds or the first alternative to reach a consensus greater than $75 \%$ of all the participants. Let us suppose that an agent have an accepting range of 0.20 , which would allow him to accept all the alternatives (according to his preferred alternative) that vary between 0.89 and 0.69 . This means that in this situation the agent would never be in conditions to accept any requests. This also means that even in case that only one more acceptance is needed for Alt2 and Alt4 to reach the 75\%, the same agent would still seek Alt1 as his only objective for that meeting. This way the agent would be losing a clear opportunity to reach a final satisfaction level of 0.54 , while only reaching a satisfaction level of 0.11 . Besides this, even if the acceptance range would allow the agent to accept Alt2, if that alternative was never requested to him, he would never consider it as an objective. Given that agents must demonstrate a social behaviour equivalent to human beings, and that they should demonstrate and generate intelligence, this does not seem to be the best approach. 
The goal of this work is to prevent these situations from happening. Therefore the main idea is to provide an agent with the ability to identify tendencies and to be able to redefine his objectives. This way we believe that it is possible to maximize the satisfaction of every agent as well as the entire group, which will result in decisions with much higher quality.

Our model is very simple and is based in the Algorithm 1 (written in pseudocode):

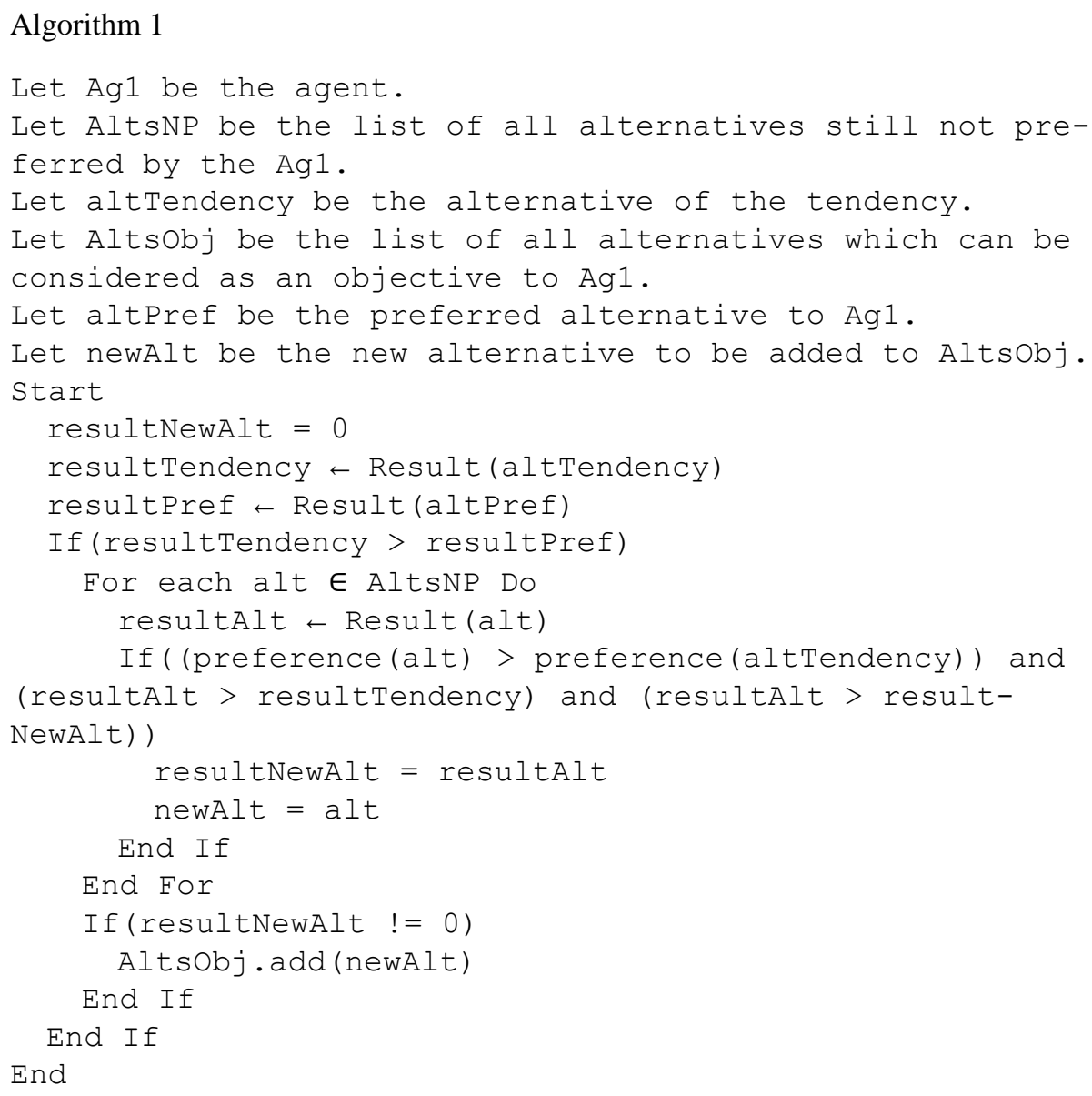

The model is based mainly in two parts. In the first part, a tendency is identified and the agent will verify if there are any conditions that allow him to add another alternative (different from his initial preferred alternatives) to his list of objectives. Every time an agent saves the knowledge about a new alternative preference for another agent he will use the formula (1) in order to measure the tendency result for that new alternative (in pseudocode it is equivalent to the Result() tag). In case he verifies that tendency already has a higher result compared to his preferred alternative (once again using formula (1)), he will analyze all the alternatives that are still not part of the list of his objectives and verify if there is any alternative with a preference greater than the tendency. From the 
list of all possible alternatives that fit this condition he will select the one which provides the highest result and add it to his list of objectives. The agent will then be able to choose that alternative for future requests.

The second part is related with how the agent chooses, from his list of objectives, which alternative should be used for a request. For that, he will use the formula that measures the 'result' and only considering alternatives from his list of objectives. It is assumed that the initial preferred alternatives by the agent are also included in his list of objectives. The formula (1) is used to measure the result for each alternative in the list of objectives.

$$
A_{\text {Result }_{A l t_{X}}}=\frac{\text { Alt }_{x} * C S+\left(\frac{N S}{N D}\right) * C O}{C S+C O}
$$

Where:

- $A l t_{x}$ is the assessment done to the alternative for which the result is being measured;

- $C S$ is the value of Concern for Self [1,2,3];

- $N S$ is the current number of agents supporting Alt $_{x}$;

- $N D$ is the total number of participating agents;

- $C O$ is the value of Concern for Others $[1,2,3]$.

In case an agent without a defined behaviour is being considered, the formula can also be used by giving the same value to $C S$ and $C O$ variables (for example " 1 ") for that agent. The alternative that provides the highest $A_{\text {Result }}$ will be chosen whenever the agent makes a new request. This formula will allow the agent to define his objectives according to the importance of the alternatives and how likely they are to be chosen at a certain time during the discussion.

\section{$3 \quad$ Evaluation and Results}

The considered scenario involves agents' negotiation in order to solve the problem of choosing a desktop monitor for an organization that wants to purchase 200 new desktop monitors to one of its subsidiaries. Each agent intends to represent one member of the organization administration board. Each alternative has been classified according to five criteria: Size (numerical, without value), Resolution (numerical, maximization), $\mathrm{Hz}$ (numerical, maximization), Ms (numerical, minimization) and Price (numerical, minimization).

In Table 1, all specifications are presented for each considered alternative.

The satisfaction and the level of consensus are used as metrics to evaluate the overall performance of the different scenarios. The satisfaction metric is used to understand the quality perception (of the decision-maker that is represented) towards the chosen alternative or the alternative supported by most agents at a certain time. For that, the notion of satisfaction that is used is the one proposed in [10]. The satisfaction is measured in two parts (for agents without a defined behaviour only the first part is considered). It is first measured objectively through the formulas (2), (3) and (4). 
Table 1. Multi-Criteria Problem

\begin{tabular}{|c|c|c|c|c|c|}
\hline Alternatives & Size & Resolution & $\mathbf{H z}$ & Ms & Price \\
\hline Asus 27" ROG SWIFT PG278Q & 27 & $2560 * 1440$ & 144 & 1 & $699,99 €$ \\
\hline BenQ 27" XL2720Z & 27 & $1920 * 1080$ & 144 & 1 & $489,00 €$ \\
\hline AOC 24" E2476VWM6 & 24 & $1920 * 1080$ & 60 & 1 & $154,90 €$ \\
\hline BenQ 24" XL2430T & 24 & $1920 * 1080$ & 144 & 1 & $399,00 €$ \\
\hline LG 27" 27MP37VQ-B & 27 & $1920 * 1080$ & 60 & 5 & $210,80 €$ \\
\hline Asus LED 21.5" VS228HR & 21,5 & $1920 * 1080$ & 60 & 5 & $129,90 €$ \\
\hline Samsung LED 22" S22C570H & 22 & $1920 * 1080$ & 60 & 5 & $179,90 €$ \\
\hline BenQ 24" LED BL2420PT & 24 & $2560 * 1440$ & 60 & 5 & $399,90 €$ \\
\hline Asus LED 24" VG248QE 144Hz 3D & 24 & $1920 * 1080$ & 144 & 1 & $288,90 €$ \\
\hline Samsung 24" Curvo LED S24E500C & 24 & $1920 * 1080$ & 60 & 4 & $199,90 €$ \\
\hline \multicolumn{6}{|c|}{$D_{\text {Lost }}=A l t_{F}-A l t_{P}$} \\
\hline \multicolumn{6}{|c|}{$A_{\text {Conversion }}=2 A l t_{F}-1$} \\
\hline$D_{\text {Sati }}$ & & Los & & & (4) \\
\hline
\end{tabular}

Where:

- $D_{\text {Lost }}$ is the loss of decision maker's satisfaction based in the difference between the assessments made for the alternative chosen by the group and for his preferred alternative. The loss is zero when the chosen alternative is the same as his preferred alternative;

- Alt $_{F}$ is the assessment made by the participant for the final alternative, alternative chosen by the group;

- $A l t_{P}$ is the assessment made by the participant for his preferred alternative;

- $A_{\text {Conversion }}$ is the conversion of the assessment made by the participant in the range [-1..1].

The second part relates the $D_{\text {Satisfaction }}$ and the behaviour defined by the decisionmaker. In this second part, the satisfaction is measured according to the values of the agent's defined behaviour (agent's with defined behaviour follow the work proposed in [21]) for concern for self and concern for others dimensions. So, the $D_{\text {Satisfaction }}$ is remeasured using formula (5).

$$
D_{\text {Satisfaction }}=\frac{D_{\text {Satisfaction }} * C S+O A A D_{\text {Satisfaction }} * C O}{C S+C O}
$$

Where:

- $C S$ is the value of Concern for Self $[1,2,3]$;

- $O A A D_{\text {Satisfaction }}$ is the average satisfaction of all the remaining agents;

- $C O$ is the value of Concern for Others $[1,2,3]$. 
The level of consensus is measured with the value of the alternative that gathered more supporters, at the time $\mathrm{t}$, during iteration $\mathrm{i}$, or round $\mathrm{r}$.

In order to evaluate our model, three simulation environments have been considered (12 Agents and 5 Alternatives; 12 Agents and 10 Alternatives; 40 Agents and 10 Alternatives). In each simulation environment, three experiments have been performed and the average satisfaction and consensus levels were measured. Each experiment was performed 100 times, in 900 simulations. For each simulation environment the information used in the configurations will be the same for the three experiments so that the results can be compared. However, these configurations (such as the agent's defined behaviour and its preferences) have been randomly generated. In the first experiment, agents are given the ability to forecast tendencies. In this first experiment, the agents use the first part of the model proposed in Section 2 (make use of Tendency Forecast but never change the requested alternatives). In the second experiment, agents use the model proposed in Section 2. This way, agents will have the ability to forecast tendencies and change their preference towards which alternative should be used in the request. In the third experiment, agents use the argumentation model without what is proposed in this work. This means that the agents do not have the ability to forecast tendencies.

In the first simulation environment, we ran 100 simulations for each of three experiments. The level of consensus achieved in the experiments of "Tendency Forecast + Request" and "Without Tendency Forecast" are very high (good). On the other hand, the level of consensus obtained by the experiment of "Tendency Forecast" is quite low. The average values of consensus for the experiments "Tendency Forecast", "Tendency Forecast + Request" and "Without Tendency Forecast" are respectively 0.36, 0.67 and 0.64 . In our point of view, the experiment of "Tendency Forecast" has the lowest average level of consensus because even though agents are capable to identify tendencies and accept new alternatives that they consider to be advantageous, they still only send requests for alternatives initially preferred by the decision-maker. In practice, agents with just "Tendency Forecast" will not make true use of their ability to analyse tendencies. Agents have social skills and if they do not report nor show or make use of their change of opinion that will reflect negatively on the achieved results. Besides this, both agents with "Tendency Forecast" and "Tendency Forecast + Request" choose the alternative that they used in the last request (before the decision-making process ended) which will lead to agents with "Tendency Forecast" never using their social skills. This situation was always verified in the three simulations environments (see Fig. 1 and Fig. 3 ), and because of that the results analysed were mainly focused in the experiments "Tendency Forecast + Request" and "Without Tendency Forecast". In these 2 experiments it was achieved very close average level of consensus, in fact, in $62 \%$ of the times the same exact level of consensus was achieved. In $25 \%$ of the times, agents with "Tendency Forecast + Request" achieved a higher level of consensus and in the remaining $13 \%$ of the times, agents "Without Tendency Forecast" achieved a higher level of consensus. The average satisfaction level obtained in the three experiments is very similar. Experiments with the same satisfaction level are differentiated by the level of consensus that is achieved. It is important to note that the satisfaction is measured according to the alternative that the agent considered as his final choice at a time $(t)$ and the 
alternative that at the same $(\mathrm{t})$ gathered the highest consensus from all the agents. Another important point is that in practice the group satisfaction always tends to value 0 . This happens mainly because only one iteration or one round is being simulated, and because of that no user reconfigurations will be made based on the information reported to the decision-maker. Therefore, this satisfaction evaluation is always related to the very first problem configuration. The average satisfaction level in the first simulation environment for "Tendency Forecast", "Tendency Forecast + Request" and "Without Tendency Forecast" are 0.09, 0.17 and 0.09 respectively. In this case, we can consider that there is a slight advantage for agents that use "Tendency Forecast + Request". One interesting fact is that agents with "Tendency Forecast + Request" and agents "Without Tendency Forecast", in 90\% of the times, have achieved a consensus towards the same alternative which can tell us that the model here presented may not be too relevant in terms of finding the "best" solution. However, agents with "Tendency Forecast + Request" obtained a higher satisfaction compared with agents "Without Tendency Forecast" in $96 \%$ of the times. This means that agents achieved usually a consensus towards the same alternative (besides sharing the same argumentation model) due to the fact that we are considering a problem with a very low complexity level (12 agents and 5 possible alternatives).

Both Fig. 1 and Fig. 2 are related to the second simulation environment, where 12 agents aim to choose an alternative from a set of 10 possible alternatives. Compared to the previous simulation environment it is clear that the complexity of the problem is much greater.

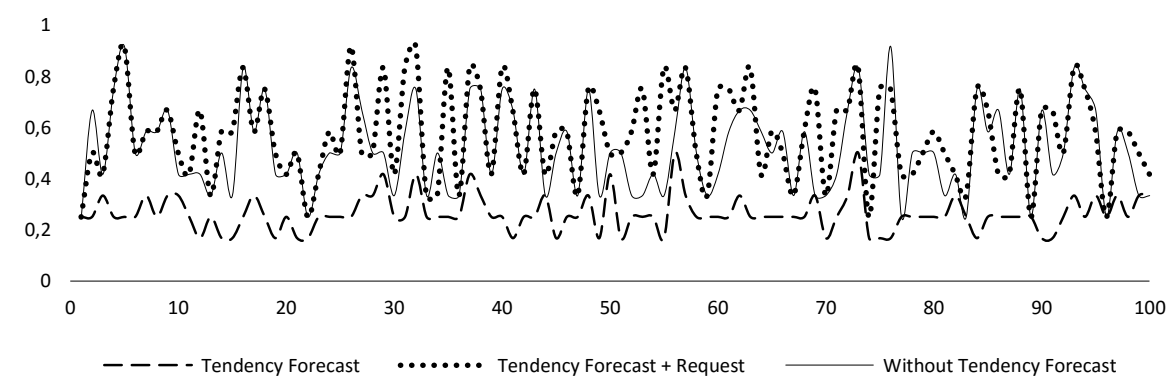

Fig. 1. Second Simulation Environment - Consensus

The level of consensus achieved by the experiments of "Tendency Forecast + Request" and "Without Tendency Forecast" is still very positive. Similar to the first simulation environment the level of consensus obtained by the experiment of "Tendency Forecast" is quite low. The average values of consensus for the experiments "Tendency Forecast", "Tendency Forecast + Request" and "Without Tendency Forecast" are 0.26, 0.57 , and 0.51 respectively. This means that compared to the first simulation environment there was a loss of $0.1,0.1$ and 0.13 respectively. This allows us to assume that not only do agents that use "Tendency Forecast + Request" achieve better results for satisfaction and level of consensus; they also have the ability to deal with more complex problems better than agents "Without Tendency Forecast". Anyway, now, this is something that needs more evidence in order to be proved and that is the reason why the 
third and last simulation environment was considered, where 40 Agents e 10 Alternatives will be used so that the problem complexity can be even greater.

In this second simulation environment, agents with "Tendency Forecast + Request" obtained the same level of consensus of agents "Without Tendency Forecast" in 53\% of the times (less 9\% compared to the first simulation environment). More importantly in this simulation environment (more complex) agents with "Tendency Forecast + Request" achieved a higher level of consensus $37 \%$ of the times against only $10 \%$ of the times where agents "Without Tendency Forecast" achieve a higher level of consensus.

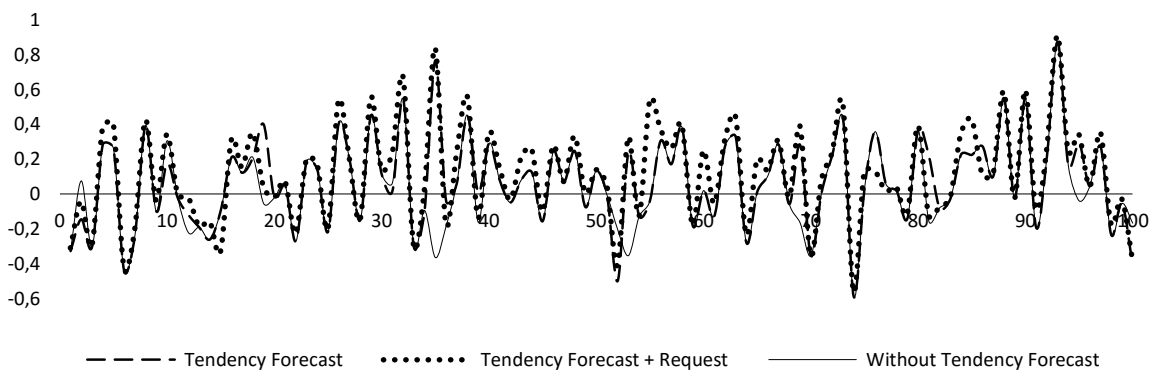

Fig. 2. Second Simulation Environment - Satisfaction

Fig. 2 shows the results obtained for the agents' average satisfaction level in the second simulation environment. Once again, the average satisfaction level obtained is very similar in both three experiments. The average satisfaction levels for "Tendency Forecast", "Tendency Forecast + Request" and "Without Tendency Forecast" are now $0.07,0.12$ e 0.04 respectively. There is still a slight remarkable advantage for agents that use "Tendency Forecast + Request". A very interesting fact compared with the first simulation environment is that now agents only achieved a consensus towards the same alternative $82 \%$ of the times. Knowing that agents that use "Tendency Forecast + Request" achieved the highest average satisfaction level, this may mean that when we are consider more complex problems agents with "Tendency Forecast + Request" will achieve better decisions. It is also important to note that in $92 \%$ agents with "Tendency Forecast + Request" achieved a higher average satisfaction level against $7 \%$ of the times where agents "Without Tendency Forecast" achieve a better average satisfaction level and only in $1 \%$ of the times the same average satisfaction level was achieved in both situations.

Both Fig. 3 and Fig. 4 are related to the last simulation environment, where 40 agents attempt to achieve a consensus for an alternative from a set of 10 possible alternatives, and therefore will be the most complex scenario from the three studied in this work.

The level of consensus achieved for the experiments of "Tendency Forecast + Request" and "Without Tendency Forecast" is $0.18,0.47$, and 0.40 respectively (Fig. 3). This means that compared to the first simulation environment there was a loss of 0.18 , 0.20 and 0.24 respectively. This allows us to assume that agents that use "Tendency Forecast + Request" obtain better results for the level of consensus in either less or more complex problems. 


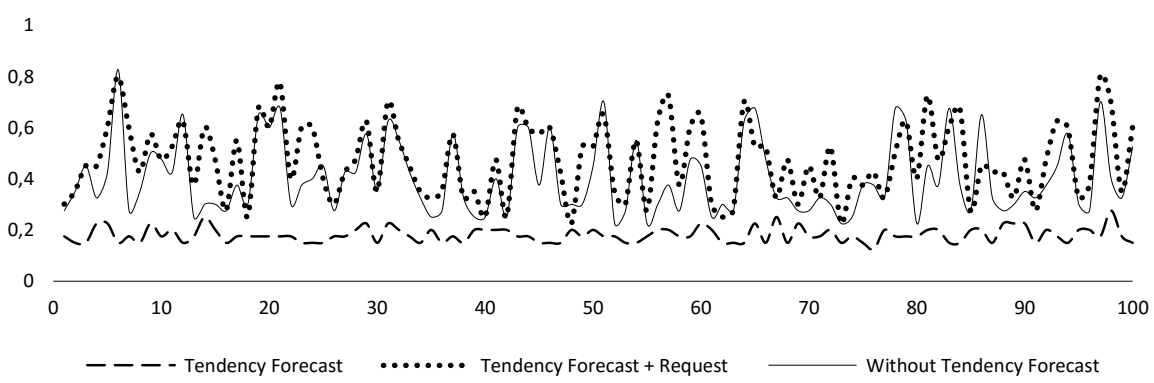

Fig. 3. Third Simulation Environment - Consensus

In this last simulation environment, agents with "Tendency Forecast + Request" obtained the same consensus level as agents "Without Tendency Forecast" in only 23\% of the times (less 39\% compared to the first simulation environment). More importantly, it is in this simulation environment (the more complex) that agents with "Tendency Forecast + Request" have achieved a level of consensus greater in $65 \%$ of the times against only $12 \%$ of the times where agents "Without Tendency Forecast" will achieve a higher level of consensus. This shows that as the problem becomes more complex agents with "Tendency Forecast + Request" will also become better at achieving higher levels of consensus and at more times compared with other agents.

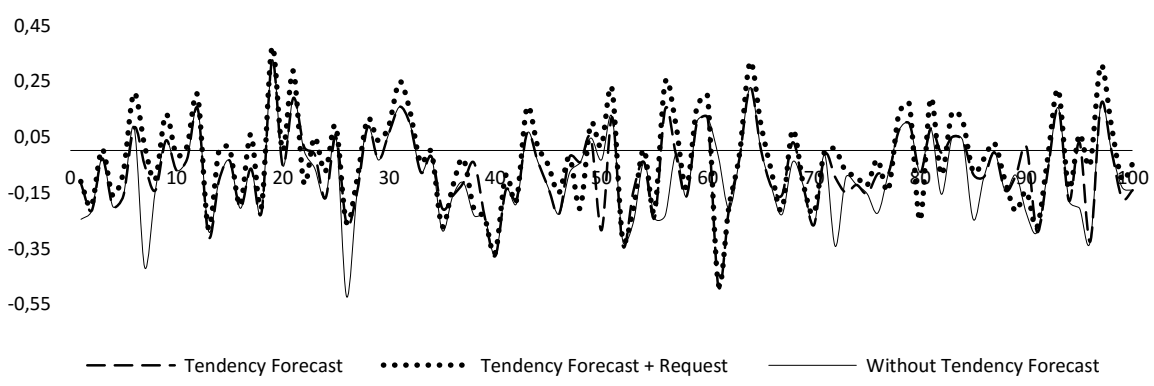

Fig. 4. Third Simulation Environment - Satisfaction

Fig. 4 shows the results for the average satisfaction levels obtained in the last simulation environment. Once again, the average satisfaction level obtained in three experiments is very similar. The average satisfaction levels obtained for "Tendency Forecast", "Tendency Forecast + Request" and "Without Tendency Forecast" are now -0.06, -0.02 and -0.09 respectively. This allows us to understand that agents that use "Tendency Forecast + Request" will always have the higher satisfaction levels, even if the problem is more or less complex. Once again, it was possible to identify a drop in the percentage where agents with "Tendency Forecast + Request" and agents "Without Tendency Forecast" achieve a consensus for the same decision, only happening $79 \%$ of the times. In $93 \%$ of the times, agents with "Tendency Forecast + Request" achieved a higher satisfaction level compared with agents "Without Tendency Forecast". 


\section{Conclusions and Future Work}

In this work, we propose a tendency analysis model with the goal to make GDSS that use negotiation models more intelligent. Our model has the main goal to improve the quality of the decision that is made as well as the group capacity to achieve a consensus. For that, agents that represent decision-makers must analyse the alternatives tendency and use the proposed algorithm to identify situations where they should reformulate their objectives.

To test our model and algorithm a case of study was performed with three different simulation environments that represent three different levels of complexity. We were able to conclude that agents that use the tendency analysis model manage to achieve in average higher levels of consensus when compared to agents that under the same circumstances do not use it. Besides this, agents that use the proposed model also manage to achieve higher levels of satisfaction. We also concluded that has the context's level of complexity increases, the tendency model becomes even more important. In the most complex simulation environment that we testes, agents with the ability to analyse tendencies were able achieve a higher consensus $65 \%$ of the times while agents that did not use this model were only able to achieve a higher consensus only $12 \%$ of the times. When measuring the level of satisfaction in the same environment agents with the ability to analyse tendencies achieved a higher level of satisfaction in $93 \%$ of the times. By combining both measures in the same study (satisfaction and consensus), it clearly shows the importance of providing agents with the ability to analyse tendencies in order to obtain decisions with higher quality in the context of this work.

As future work, we intend to expand our model. More precisely, we want to include in our model the analysis of credibility. Credibility (in a very simple way) can be important for situations when a decision-maker considers another to be credible it might make sense to support his opinion even if that was not part of initial preferences. This way we think to be possible (together with automatic negotiation mechanisms) to achieve solutions with more quality as well as with higher levels of consensus, with the system always informing the decision-maker properly about each step of the negotiation process and the reasons behind suggestions that are given to him.

\section{Acknowledgements}

This work has been supported by COMPETE Programme (operational programme for competitiveness) within project POCI-01-0145-FEDER-007043, by National Funds through the FCT - Fundação para a Ciência e a Tecnologia (Portuguese Foun-dation for Science and Technology) within the Projects UID/CEC/00319/2013, UID/EEA/00760/2013, and the João Carneiro $\mathrm{PhD}$ grant with the reference SFRH/BD/89697/2012 and by Project MANTIS - Cyber Physical System Based Proactive Collaborative Maintenance (ECSEL JU Grant nr. 662189). 


\section{References}

1. Luthans, F.: Organizational behavior. McGraw-Hill, Boston (2005)

2. DeSanctis, G., Gallupe, B.: Group decision support systems: a new frontier. ACM SIGMIS Database 16, 3-10 (1984)

3. Desanctis, G., Gallupe, R.B.: A foundation for the study of group decision support systems. Management science 33, 589-609 (1987)

4. Grudin, J.: Group dynamics and ubiquitous computing. Communications of the ACM 45, 74-78 (2002)

5. Marreiros, G., Santos, R., Ramos, C., Neves, J.: Context-Aware Emotion-Based Model for Group Decision Making. Intelligent Systems, IEEE 25, 31-39 (2010)

6. Carneiro, J., Santos, R., Marreiros, G., Novais, P.: UbiGDSS: A Theoretical Model to Predict Decision-Makers' Satisfaction. International Journal of Multimedia and Ubiquitous Engineering 10, 191-200 (2015)

7. Kwon, O., Yoo, K., Suh, E.: UbiDSS: a proactive intelligent decision support system as an expert system deploying ubiquitous computing technologies. Expert systems with applications 28, 149-161 (2005)

8. Shim, J.P., Warkentin, M., Courtney, J.F., Power, D.J., Sharda, R., Carlsson, C.: Past, present, and future of decision support technology. Dec. support systems 33, 111-126 (2002)

9. Paul, S., Seetharaman, P., Ramamurthy, K.: User satisfaction with system, decision process, and outcome in GDSS based meeting: an experimental investigation. In: Proceedings of the Proceedings of the 37th Annual Hawaii International Conference on System Sciences (HICSS'04)-Track 1-Volume 1, pp. 10037.10032. IEEE Computer Society, (2004)

10. Carneiro, J., Marreiros, G., Novais, P.: Using Satisfaction Analysis to Predict Decision Quality. International Journal of Artificial Intelligence ${ }^{\mathrm{TM}}$ 13, 45-57 (2015)

11. Muller, J., Hunter, A.: An argumentation-based approach for decision making. In: Tools with Artificial Intelligence (ICTAI), 2012 IEEE 24th International Conference on, pp. 564571. IEEE, (2012)

12. Marey, O., Bentahar, J., Khosrowshahi-Asl, E., Sultan, K., Dssouli, R.: Decision making under subjective uncertainty in argumentation-based agent negotiation. Journal of Ambient Intelligence and Humanized Computing 6, 307-323 (2015)

13. Dennis, A.R.: Information exchange and use in small group decision making. Small Group Research 27, 532-550 (1996)

14. Hill, G.W.: Group versus individual performance: Are $\mathrm{N}+1$ heads better than one? Psychological bulletin 91, 517 (1982)

15. Higgins, E.T.: Making a good decision: value from fit. Am. Psychologist 55, 1217 (2000)

16. Schimmack, U., Oishi, S., Furr, R.M., Funder, D.C.: Personality and life satisfaction: A facet-level analysis. Personality and Social Psychology Bulletin 30, 1062-1075 (2004)

17. Judge, T.A., Heller, D., Mount, M.K.: Five-factor model of personality and job satisfaction: a meta-analysis. Journal of applied psychology 87, 530 (2002)

18. Ramchurn, S.D., Jennings, N.R., Sierra, C.: Persuasive negotiation for autonomous agents: A rhetorical approach. (2003)

19. Ito, T., Shintani, T.: Persuasion among agents: An approach to implementing a group decision support system based on multi-agent negotiation. In: International Joint Conference on Artificial Intelligence, pp. 592-599. Citeseer, (1997)

20. Kraus, S., Sycara, K., Evenchik, A.: Reaching agreements through argumentation: a logical model and implementation. Artificial Intelligence 104, 1-69 (1998)

21. Martinho, D., Carneiro, J., Marreiros, G., Novais, P.: Dealing with Agents' Behaviour in the Decision-Making Process. In: SOOW. (2015) 